\title{
Onycholysis is not a negligible malaise especially in diabetics and people suffering from psoriasis: a dignified way to treat the concern with cuminum and cardamom
}

\section{Piotr Brzeziński', Lorenzo Martini ${ }^{1,2}$}

${ }^{1}$ University of Siena, Department of Pharmaceutical Biotechnologies, Via A. Moro 2, 53100 Siena, Italy, ${ }^{2}$ C.R.I.S.M.A. Inter

University Centre for Researched Advanced Medical Systems

Corresponding author: Prof. Lorenzo Martini, E-mail: martini36@unisi.it

Sir,

Onycholysis is the medical term for when nail separates from the skin underneath it. Onycholysis is not uncommon, and it has several possible causes.

This condition lasts for several months, because a fingernail or toenail won't reattach to its nail bed. Once a new nail grows to replace the old one, the symptoms should resolve. Fingernails take 4 to 6 months to fully regrow, and toenails can take 8 to 12 months $[1,2]$.

Injury to the nail can cause onycholysis. Wearing tight shoes can cause injury. The condition can also result from an allergy to products used on the nail, like chemical nail polish remover or artificial nail tips. Onycholysis can also be a symptom of nail fungus or psoriasis [3].

Other causes include a reaction to a systemic medication or trauma. Even repetitive tapping or drumming of the fingernails can count as trauma.

Nails tend to be a barometer of the overall health. If the nails look unhealthy or have problems like onycholysis, this could be the first visible sign that something deeper is going on in human body [4].

Sometimes onycholysis can indicate a serious yeast infection or thyroid disease. It can also mean that the person who suffers from this nail disease is not getting enough of essential vitamins or minerals, such as iron.
Amongst the symptoms of onycholysis, the nail will begin to peel upward off of the nail bed underneath. This is not usually painful while it happens. The affected nail may become yellow, greenish, purple, white, or gray, depending on the cause.

Determining the cause of your onycholysis is the most important step. Once the cause is found, treating the underlying issue will help the nail lifting resolve.

While it is important to keep the nails short, aggressive clipping is not recommended. As the affected portion of the nail grows out, the patient will be able to clip off the lifted nail as the new nail continues to come in.

As far as treating the underlying condition the cause of the nail separation will need to be addressed before the symptoms stop occur.

Onycholysis, especially recurring onycholysis, might need a diagnosis and a prescription in order to heal.

It's not uncommon to have onycholysis as a symptom of psoriasis. The Psoriasis and Psoriatic Arthritis Association estimates at least 50 percent of people with psoriasis experience problems with their nails.

Fingernails in particular are affected by psoriasis. Treating psoriasis in the nails can be difficult. Doctors may prescribe topical vitamin $\mathrm{D}$ or corticosteroids to treat nail psoriasis.

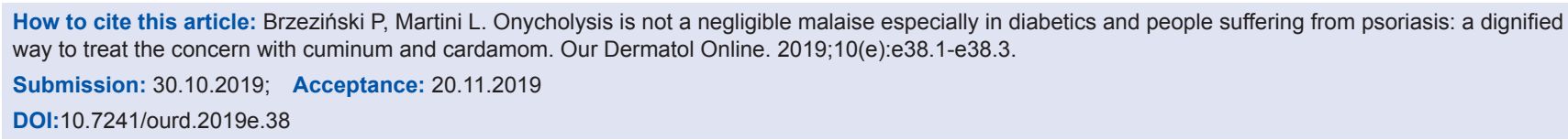


A blood test may reveal that the patient has a thyroid condition or vitamin deficiency causing him to have onycholysis. In this case, the physician may prescribe medication or an oral supplement to treat the underlying cause of the onycholysis.

In the meantime, the subject who suffers from onycholysis might want to try to treat his onycholysis at home. It's better not to try to clean underneath the nail, as that could make the problem worse or sweep bacteria deeper underneath the nail.

A 2013 study Trusted Source showed that tea tree oil can help treat fungus and yeast infections that happen underneath the nail. Applying a mixture of tea tree oil diluted by a carrier oil, such as jojoba oil or coconut oil, may get rid of the fungus. Make sure to keep the nail dry while it heals.

As far as preventing the occurrence of onycholysis, this can result from trusted source a skin sensitivity to products such as glue, acrylics, or acetone that are used during manicures and pedicures. If the patient has skin allergies to these products, he/she should avoid the nail salon. It is always better to choose allergen-free products and paint the nails at home.

Artificial "tips" applied to the nail can also cause trauma of the nail bed, with onycholysis as the result.

If the patient has a fungus or yeast growth causing onycholysis, he/she can stop it from spreading by taking proper care of his/her nails. It is always better not to bite nails, as this will spread the problem from nail to nail and may possibly affect even the mouth.

If onycholysis is happening in toenails, it is advisable to make sure the patient does wear clean socks and expose the feet to dry air for as much of the day as possible.

The AA, aware that tea tree oil mixed with coconut or jojoba oil, according to trivial medicine, is an excellent remedy to treat this nail disease, have decide to use a combination of the chief biological principles contained in tea tree oil and solvents (oils) that contain the same fatty acids of coconut or jojoba oil and for this specific reason they have chosen this cosmetic mix:

Wild thyme extract [5].

Elettaria cardamomum seed extract (containing 50\% of myristic acid and 20\% of lauric acid) [6].
Cuminum cyminum extract (extremely rich in p-cymene) $(0,4 \%$, that corresponds to the maximum percentage admitted by Annex III) [7].

Diluted in: Palm kernel oil (rich in lauric acid); Palm oil (rich myristic acid); Babassu seed oil (babassu palm (Attalea speciosa) rich in myristic acid.

The AA have used the cosmetic system (an oily liquid emulsion to apply with a cotton pad) on a volunteer (86 y. old) suffering from diabetes type I and psoriatic rheumatoid arthritis.

They payed attention to moisten even the interstice between the nail and the nail bed where debris may burrow.

The volunteer used to take supplements of vit. D and apply a lipogel containing ergocalciferol and pomades containing hydrocortisone acetate on the big toe to combat psoriasis, but the AA decided to stop the administration both of vit $\mathrm{D}$ and corticosteroids for all the duration of the treatment.

She did not suffer from fungal infections, since no manifestations of onychomycosis were evident.

The AA administered pills of ferrous gluconate (114 mg 3 times/day) as complementary dietary supplementation.

The treatment lasted 2 weeks.

The big toe nail begins to reattach the nail bed after the $6^{\text {th }}$ day and the purple colour of the nail grows yellowish after th $7^{\text {th }}$ day and whitish after the $11^{\text {st }}$ day.

As the AA wrote before, no symptom of pain is evident and so the only signals of remission of the disease is the change of colour of the nail and the diminishing of the interstice between the nail and the nail bed.

\section{Consent}

The examination of the patient was conducted according to the Declaration of Helsinki principles.

\section{REFERENCES}

1. Tatu AL, Voicu C, Clatici VG. Therapeutic management of a green nail syndrome -Pseudomonas aeruginosa and Tricophyton tonsurans coinfection detected by mass spectrometry. RoJCED. 2016;3-4:167-9.

2. Yorulmaz A, Yalcin B. A novel dermoscopic feature in traumatic 
onycholysis. Our Dermatol Online. 2018;9:307-9.

3. Tamer F, Yuksel ME. Onychomycosis due to mixed infection with non-dermatophyte molds and yeasts. Our Dermatol Online. 2019;10:267-9.

4. Aqil N, Nassiri A, Baybay H, Douhi Z, Elloudi S, Mernissi FZ. Erythroderma: A clinical and etiological study of 92 patients. Our Dermatol Online. 2019;10:1-6.

5. Rehman AU, Mannan A, Inayatullah S, Akhtar Z. Biological evaluation of wild thyme (Tymus serpyllum). Pharmaceut Biol. 2009;47:628-33.

6. Ashokkumar K, Murugan M, Dhanya MK, Warkentin TD. Botany, traditional uses, phytochemistry and biological activities of cardamom [Elettaria cardamomum (L.) Maton] - A critical review.
J Ethnopharmacol. 2020;246:112244.

7. Hada M, Nishi K, Ishida M, Onda H, Nishimoto S, Sugahara T. Inhibitory effect of aqueous extract of Cuminum cyminum $\mathrm{L}$. seed on degranulation of RBL-2H3 cells and passive cutaneous anaphylaxis reaction in mice. Cytotechnology. 2019;71:599-609.

Copyright by Piotr Brzeziński, et al. This is an open-access article distributed under the terms of the Creative Commons Attribution License, which permits unrestricted use, distribution, and reproduction in any medium, provided the original author and source are credited.

Source of Support: Nil, Conflict of Interest: None declared. 